\title{
ГЕОГРАФИЯ
}

УДК 551.58

\section{ИЗМЕНЕНИЯ ИНСОЛЯЦИИ, ВЫЗВАННЫЕ ПЛАНЕТНЫМИ ВОЗМУЩЕНИЯМИ ОРБИТЫ ЗЕМЛИ И ВАРИАЦИЕЙ СОЛНЕЧНОЙ ПОСТОЯННОЙ}

\author{
М.Б. Богданов, А.В. Катрущенко, А.Н. Сурков \\ Саратовский государственный университет, \\ кафедра метеорологии и климатологии \\ E-mail: BogdanovMB@info.sgu.ru
}

С использованием современных численных методов небесной механики рассчитан приходящий к Земле поток излучения Солнца в предположении неизменности его светимости на интервале времени с 1700 по 2000 г. В спектре мощности временного ряда инсоляции, наряду с гармониками годичного периода обращения Земли, четко проявляются гармоники синодических периодов планет Венеры, Марса, Юпитера и Сатурна, а также гармоники, отвечающие комбинационным частотам. Их периоды заключены в интервале от 100 дней до 15.7 года. Основными гармониками спектра мощности являются гармоника, соответствующая синодическому периоду Юпитера 399d.0, и первая гармоника синодического периода Венеры c периодом 291d.9. Их амплитуды приблизительно равны и составляют около 40 мВт/м². Рассчитан также спектр мощности временного ряда реальных изменений инсоляции в 1978-2005 гг., вызванных как планетными возмущениями, так и вариацией солнечной постоянной. Наряду с 11-летним циклом солнечной активности в этом спектре прослеживается и влияние планетных гармоник.

Changes in the Insolation, Caused by the Planetary Perturbations of the Earth's Orbit and by Variation in the Solar Constant

\section{M.B. Bogdanov, A.V. Katrushchenko, A.N. Surkov}

With the use of modern numerical methods of celestial mechanics, the incoming irradiance flux to the Earth from the Sun has been calculated under the assumption of the invariability of its luminosity through interval of 1700-2000 A.D. Harmonics of the annual period of the Earth as well as harmonics of the synodic period of Venus, Mars, Jupiter, Saturn and harmonics corresponded to combination frequencies, are clearly visible in the power spectrum of time series of the insolation. The periods of these harmonics are located in the range from 100 days to 15.7 years. The main harmonics in the power spectrum are corresponded to the synodic period of Jupiter 399d.0, and the first harmonic of the synodic period of Venus with period 291d.9. Their amplitudes are approximately equal to $40 \mathrm{~mW} / \mathrm{m}^{2}$. The power spectrum of time series of real changes of the insolation during 1978-2005, which was caused by the planetary perturbations and variation in the solar constant, has been also calculated. Influence of planetary harmonics was also observed in this spectrum together with the 11-year cycle of the solar activity.

\section{Введение}

Поток солнечного излучения является основным фактором, определяющим климат Земли и процессы, протекающие в ее атмосфере и гидросфере. Как показали проведенные исследования [1-4], из-за наличия специфических обратных связей глобальный климат весьма чувствителен к изменениям инсоляции. Вариация среднего потока на $1.0 \mathrm{BT} / \mathrm{m}^{2}$ вызывает изменение средней глобальной температуры на $0.10 \mathrm{~K}$, что в два раза превышает ожидаемое значение для равновесной температуры абсолютно черного тела [5]. Поэтому исследование возможных изменений инсоляции представляет большой интерес для всего комплекса наук, изучающих нашу планету.
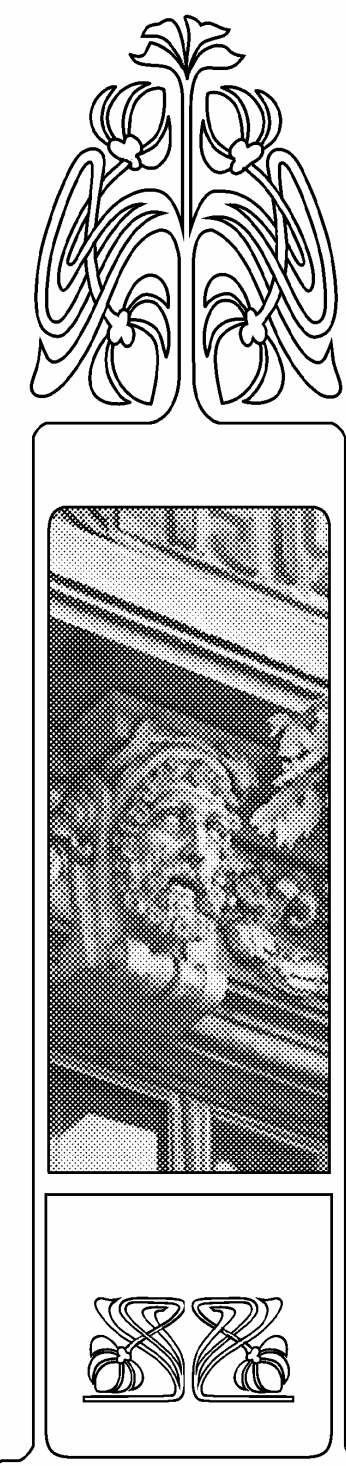

\section{HАУЧHЫĬ OTAEN}

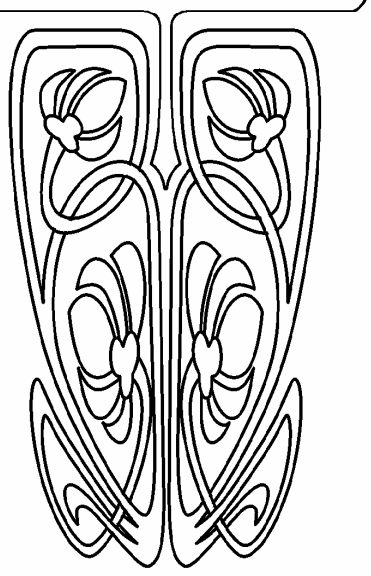


Как известно, изменение инсоляции вызывается двумя причинами: геометрической изменением расстояния от Земли до Солнца, и вариацией светимости Солнца, проявляющейся в изменении солнечной постоянной. В последние десятилетия вопросы изменения солнечной постоянной интенсивно изучаются, в том числе и с использованием высокоточных внеатмосферных наблюдений. Результаты ранних исследований представлены в монографии [6], а более поздние данные рассмотрены в статьях $[7,8]$.

Наиболее заметный вклад в изменение инсоляции вносит орбитальное движение Земли. Различие в величине потока для перигелия и афелия составляет около $6.7 \%$. Возмущения орбиты Земли, вызванные притяжением других тел Солнечной системы, способны заметным образом перераспределять суммарную инсоляцию полушарий. М. Миланковичем [9] впервые было показано, что вызываемые этими возмущениями изменение угла наклона плоскости эклиптики к плоскости небесного экватора, движение перигелия земной орбиты и изменение ее эксцентриситета приводят к изменениям климата северного и южного полушарий на временной шкале в десятки и сотни тысяч лет. Результаты более поздних исследований данной проблемы рассмотрены в монографии А.С. Монина [10] и сборнике [11]. Очевидно, что планетные возмущения должны вызывать и короткопериодические изменения инсоляции с характерным временем от сотен дней до нескольких лет. Недавно внимание вновь было привлечено к этой проблеме [12]. Однако полученные в ней оценки являются весьма грубыми. Современный уровень развития небесной механики позволяет получить строгое решение данной задачи.

Целью настоящей работы является оценка частот и амплитуд короткопериодических гармоник изменения инсоляции, вызванных планетными возмущениями орбиты Земли, а также изучение реально наблюдаемых изменений инсоляции, обусловленных совместным действием планетных возмущений и вариации солнечной постоянной.

\section{Расчет величины инсоляции} с учетом планетных возмущений

До последней четверти XX века координаты планет Солнечной системы рассчиты- вались на основе аналитических теорий. Однако ряд трудностей, и в частности большие погрешности оценок расстояний [13], заставили отказаться от их применения. Начиная с 1976 г., по решению Международного астрономического союза (IAU), описание движения тел Солнечной системы проводится с использованием численного интегрирования дифференциальных уравнений движения при учете возмущений от всех массивных тел (включая и ряд астероидов), а также релятивистских эффектов. Для решения нашей задачи мы использовали доступный в сети Интернет генератор эфемерид HORIZONS (http://ssd.jpl.nasa.gov/cgi-bin/eph/). Он разработан Лабораторией реактивного движения HACA (JPL, NASA) на основе численной теории DE-406/LE-406 и позволяет рассчитывать положение центра массы планеты с погрешностью [14], не превышающей 25 м. Такая точность более чем достаточна для наших целей.

В качестве оценки величины мгновенной инсоляции мы приняли мощность потока солнечной радиации $I(t)$, приходящего на площадку в 1 кв. м., ориентированную перпендикулярно направлению на Солнце и расположенную от него на расстоянии центра массы Земли $r(t)$ в заданный момент времени $t$. Для величины солнечной постоянной $Q$ использовалось значение $1366.22 \mathrm{Bт} / \mathrm{m}^{2}$, принятое в качестве космического абсолютного радиометрического эталона (SARR). Величина инсоляции рассчитывалась по формуле

$$
I(t)=Q r^{-2}(t),
$$

в которой расстояние между центрами масс Солнца и Земли $r(t)$ измерялось в астрономических единицах.

С целью дальнейшего анализа мы рассчитали временной ряд инсоляции в интервале с 1.01.1700 г. по 31.12.2000 г. с дискретностью $10^{\mathrm{d}}$. Полное число отсчетов ряда равнялось 10994. Выбранная длина ряда достаточно велика для того, чтобы обеспечить хорошее частотное разрешение при спектральном анализе, и в то же время мала по сравнению с продолжительностью циклов Миланковича. Кроме того, с начала XVIII в. для некоторых пунктов уже имеются ряды инструментальных измерений метеорологических 
параметров, которые могут в дальнейшем быть сопоставлены с рядом изменения инсоляции.

\section{Спектральный анализ временного ряда инсоляции}

Поскольку значения инсоляции рассчитывались с использованием высокоточной численной теории, то для поиска короткопериодических гармоник в данном временном ряде естественно применить методику цифрового спектрального анализа. Мы выбрали классический вариант спектрального анализа с расчетом спектра мощности путем фурьепреобразования автокорреляционной функции [15], так как он позволяет получать надежные оценки разрешающей способности и доверительного интервала.

Первоначально была проведена фильтрация ряда с целью уменьшения влияния годичного движения Земли. В качестве основного периода был выбран аномалистический год (промежуток времени между двумя последовательными прохождениями Землей перигелия, 365. $\left.{ }^{\mathrm{d}} 25964\right)$. Амплитуда 45.8308 Вт/м² и фаза 1.62035 рад синусоиды с этим периодом были определены методом наименьших квадратов. После вычитания синусоиды из отсчетов ряда инсоляции аналогичным обра- зом были найдены параметры первой гармоники аномалистического года с периодом 182. ${ }^{\mathrm{d}} 62982$. Ее амплитуда и фаза равны соответственно $0.960628 \mathrm{Bт} / \mathrm{M}^{2}$ и 1.66957 рад. Остаточное среднеквадратичное отклонение временного ряда инсоляции б после вычитания двух основных гармоник оказалось равным 0.106430 Вт/м².

Для отфильтрованного временного ряда инсоляции были рассчитаны автокорреляционная функция и спектр мощности $S(f)$. При выбранном максимальном сдвиге автокорреляции в 9000 отсчетов для использованного корреляционного окна Ханна ширина спектрального окна составляет $\Delta f=1.481 \times 10^{-5} \mathrm{~d}^{-1}$. Число степеней свободы спектральной оценки равно 3, а длина 90\%-ного логарифмического доверительного интервала 1.35. График логарифма спектра мощности в интересующем нас диапазоне частот $f$ от нуля до $0.01 \mathrm{~d}^{-1}$ показан на рис. 1. Для его построения использовано 5001 значение спектра. Вертикальным отрезком на рисунке показан логарифмический доверительный интервал. Следует отметить, что реальное движение Земли происходит по незамкнутой орбите и является непериодическим, поэтому фильтрация двух гармоник не позволяет полностью ис-

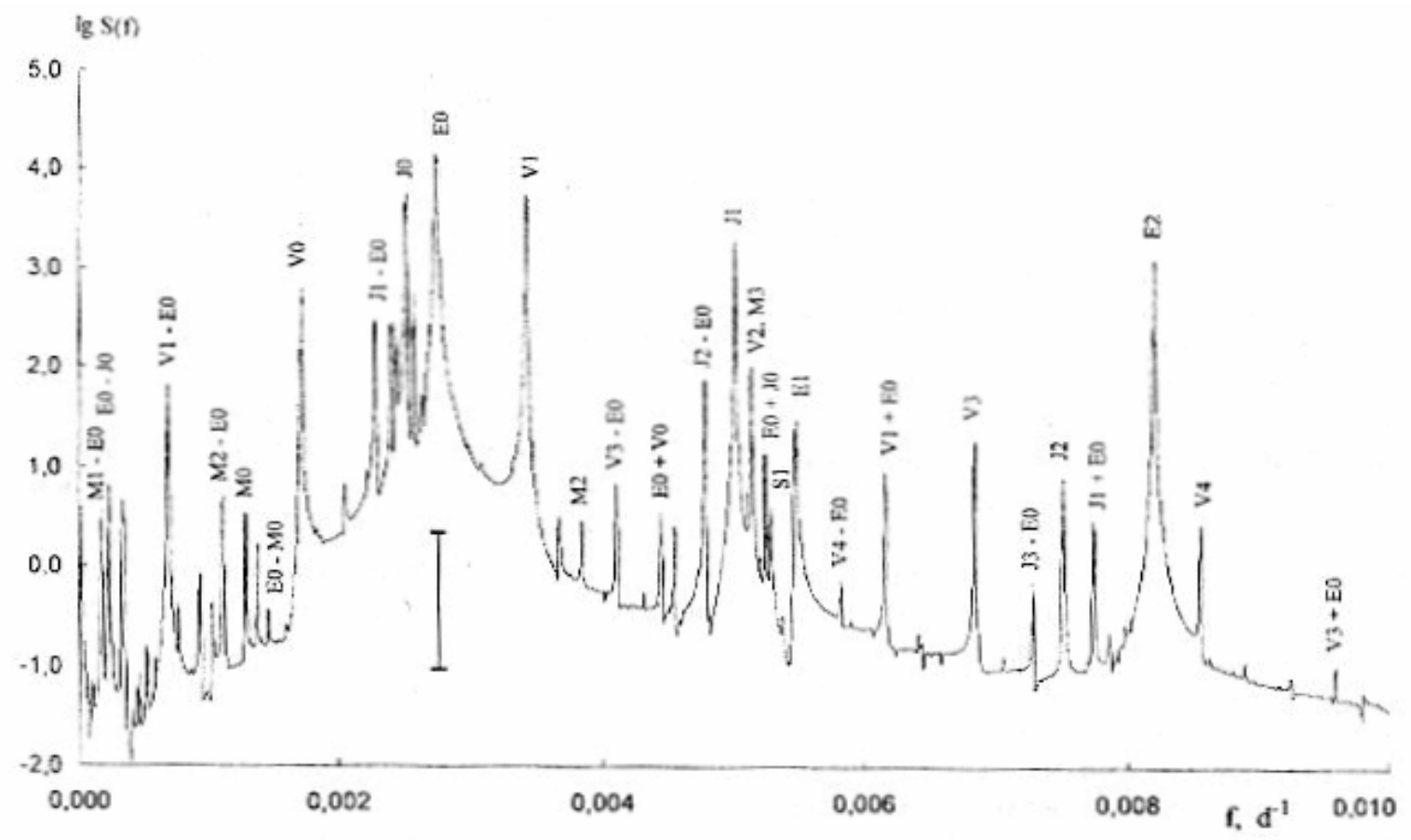

Рис. 1. График логарифма спектра мощности временного ряда изменения инсоляции, вызванного планетными возмущениями орбиты Земли 
ключить влияние годичного движения. Соответствующие пики, обозначенные символами E0 и E1, продолжают оставаться хорошо заметными в спектре мощности. Однако проведенная фильтрация сильно подавляет мощность на частотах этих гармоник и позволяет выявить множество спектральных пиков, связанных с планетными возмущениями земной орбиты.

Совмещая середину логарифмического доверительного интервала с основанием пиков, можно убедиться, что большинство гармоник являются статистически значимыми. Следует отметить, что величина доверительного интервала оценена в предположении того, что исходный временной ряд является реализацией гауссовского случайного процесса. В нашем случае изменения инсоляции детерминированы, причем уровень случайного шума близок к нулю (относительная погрешность оценки $I(t)$ порядка $\left.10^{-9}\right)$. Высокое спектральное разрешение и острые вершины пиков в спектре мощности позволяют оценивать частоты гармоник с погрешностью $\pm 0.1 \times 10^{-5} \mathrm{~d}^{-1}$, что дает возможность однозначно провести их идентификацию. Совпадение частот гармоник с ожидаемыми значениями говорит о том, что практически все они являются реальными.

Нелинейная зависимость инсоляции от расстояния (1) позволяет ожидать наличия в спектре мощности гармоник годичного периода Земли, гармоник синодических периодов планет (соответствующих разности угловых скоростей Земли и планеты) и гармоник, отвечающих комбинационным частотам. Идентификация гармоник показана у вершин пиков на рис. 1 набором букв и цифр. Буква является первой буквой английского наименования планеты, а цифра определяет номер гармоники (ноль соответствует основному периоду). Таким образом, J0 - гармоника, соответствующая синодическому периоду Юпитера, М2 - вторая гармоника синодического периода Марса, а V1 - E0 - комбинационная гармоника, частота которой является разностью частоты первой гармоники синодического периода Венеры и частоты, соответствующей аномалистическому году Земли. В рассчитанном спектре мощности, наряду с гармониками годичного периода, выявлены гармоники, связанные с планетами Венера, Марс, Юпитер и Сатурн.

Обычно при проведении спектрального анализа частоты гармоник определяются заметно точнее, чем их амплитуды. В нашем случае погрешность оценки амплитуды связана не с влиянием случайного шума, а с наложением соседних гармоник и неопределенностью уровня основания пиков в спектре мощности. В качестве оценки амплитуды гармоники нами бралась величина

$$
A\left(f_{m}\right)=\sigma \sqrt{2 \Delta f\left(S\left(f_{m}\right)-S_{0}\right)},
$$

где $\sigma-$ среднеквадратичное отклонение временного ряда; $\Delta f$ - ширина спектрального окна; $S\left(f_{m}\right)$ - значение спектральной плотности в максимуме пика; $S_{0}-$ величина спектральной плотности у основания пика, оцениваемая как полусумма значений $S(f)$, измеренных слева и справа от пика. Если принять, что погрешность оценки амплитуды определяется неопределенностью уровня основания пика, то в качестве ее верхнего предела можно взять величину

$$
\Delta A=\sigma \sqrt{2 \Delta f S_{0}} .
$$

Характеристики отождествленных гармоник, амплитуды которых превышают $1.0 \mathrm{MBT} / \mathrm{M}^{2}$, приведены в таблице. Значения периодов $T$ (в средних солнечных сутках) рассчитаны как величины, обратные измеренным частотам $f$. Поскольку значения синодических периодов планет известны с большой точностью, при необходимости периоды гармоник могут быть легко уточнены. Как видно из таблицы, наиболее заметными в спектре ряда инсоляции являются две гармоники: J0 и V1. Их амплитуды приблизительно равны и примерно в два раза превышают амплитуды следующей по мощности первой гармоники синодического периода Юпитера J1 и второй гармоники орбитального периода Земли Е2. Гармоника с номером 17 является блендой в пределы спектрального окна попадают одновременно две гармоники V2 и M3.

Спектральный анализ не дает информации о фазах гармоник. Тем не менее очевидно, что взаимное влияние гармоник J0 и V1 должно вызывать биения инсоляции с их разностной частотой. Таким образом, с периодом $1089^{\mathrm{d}} .2$ или 2.982 года амплитуды 
гармоник будут суммироваться. Но даже если время реакции обратных связей, определяющих чувствительность нашей планеты к изХарактеристики гармоник,

возникающих в спектре мощности изменения инсоляции под влиянием планетных возмущений орбиты Земли

\begin{tabular}{|c|c|c|c|c|c|}
\hline № & $f, 10^{-3} d^{-1}$ & $T, d$ & $A(f), \mathrm{MBт} / \mathrm{M}^{2}$ & $\Delta A, \mathrm{мB} / \mathrm{M}^{2}$ & $\begin{array}{c}\text { Иденти- } \\
\text { фикация }\end{array}$ \\
\hline 1 & 0.174 & 5750 & 1.11 & 0.13 & $\mathrm{M} 1-\mathrm{E} 0$ \\
2 & 0.230 & 4350 & 1.44 & 0.14 & $\mathrm{E} 0-\mathrm{J} 0$ \\
3 & 0.688 & 1450 & 4.75 & 0.19 & $\mathrm{~V} 1-\mathrm{E} 0$ \\
4 & 1.108 & 902.5 & 1.31 & 0.19 & $\mathrm{M} 2-\mathrm{E} 0$ \\
5 & 1.282 & 780.0 & 1.05 & 0.20 & $\mathrm{M} 0$ \\
6 & 1.712 & 584.1 & 14.61 & 0.59 & $\mathrm{~V} 0$ \\
7 & 2.276 & 439.4 & 10.27 & 1.15 & $\mathrm{~J} 1-\mathrm{E} 0$ \\
8 & 2.398 & 417.0 & 9.63 & 1.80 & $\mathrm{~V} 2-\mathrm{E} 0$ \\
9 & 2.506 & 399.0 & 44.06 & 3.19 & $\mathrm{~J} 0$ \\
10 & 2.564 & 390.0 & 13.38 & 2.40 & $\mathrm{M} 1$ \\
11 & 2.645 & 378.1 & 3.42 & 2.65 & $\mathrm{~S} 0$ \\
12 & 3.426 & 291.9 & 42.72 & 1.12 & $\mathrm{~V} 1$ \\
13 & 4.112 & 243.2 & 1.46 & 0.41 & $\mathrm{~V} 3-\mathrm{E} 0$ \\
14 & 4.450 & 224.7 & 1.04 & 0.36 & $\mathrm{~V} 0+\mathrm{E} 0$ \\
15 & 4.782 & 209.1 & 5.07 & 0.29 & $\mathrm{~J} 2-\mathrm{E} 0$ \\
16 & 5.014 & 199.4 & 25.46 & 0.66 & $\mathrm{~J} 1$ \\
17 & 5.138 & 194.6 & 5.93 & 0.75 & $\mathrm{~V} 2, \mathrm{M} 3$ \\
18 & 5.246 & 190.6 & 2.11 & 0.50 & $\mathrm{E} 0+\mathrm{J} 0$ \\
19 & 5.290 & 189.0 & 1.05 & 0.48 & $\mathrm{~S} 1$ \\
20 & 6.162 & 162.3 & 1.71 & 0.25 & $\mathrm{E} 0+\mathrm{V} 1$ \\
21 & 6.850 & 146.0 & 2.50 & 0.20 & $\mathrm{~V} 3$ \\
22 & 7.522 & 132.9 & 1.66 & 0.18 & $\mathrm{~J} 2$ \\
23 & 8.214 & 121.7 & 20.95 & 0.14 & $\mathrm{E} 2$ \\
\hline
\end{tabular}

менению инсоляции [5], окажется меньше этого периода, то ожидаемое изменение глобальной температуры не превысит $0.01 \mathrm{~K}$.

Наряду с рассмотренным геометрическим фактором, на изменение инсоляции существенным образом влияет и изменение солнечной постоянной. Очевидно, что учет изменения светимости Солнца не приведет к потере каких-либо выявленных гармоник, но может добавить новые пики в спектре мощности.

Временной ряд инсоляции с учетом вариации солнечной постоянной и расчет его спектра мощности

Для изучения реально наблюдаемых изменений инсоляции, происходящих под влиянием возмущений орбиты Земли и изменения солнечной постоянной, мы использовали композитный временной ряд измерения солнечной постоянной радиометром ACRIM с различных космических аппаратов. Вопро- сы погрешности измерений и методика редукции рядов данных к единой системе рассмотрены в работе [7]. Данные наблюдений были получены по сети Интернет с сервера проекта ACRIM (http://www.acrim.com/). Они представляют собой ежедневные отсчеты величины $Q$ с 17.11 .1978 г. по 21.03.2005 г. Отдельные отсутствующие значения, достаточно случайно распределенные на этом интервале времени и составляющие в совокупности около 6\% от общего числа отсчетов, были рассчитаны нами с помощью линейной интерполяции данных наблюдений. Полное число отсчетов временного ряда составило 9626.

Измеренные значения солнечной постоянной приведены к расстоянию в 1 a.e. Для получения реальных значений потока солнечного излучения нами использовалась формула (1), входящие в нее величины расстояний, как и ранее, рассчитывались с помощью генератора эфемерид HORIZONS. Из полученного временного ряда вновь были вычтены синусоида с периодом аномалистического года (с амплитудой $45.72669 \mathrm{BT} / \mathrm{m}^{2}$ и фазой 0.751951 рад) и ее первая гармоника (с амплитудой $0.953634 \mathrm{BT} / \mathrm{M}^{2}$ и фазой 0.069844 рад), параметры которых определялись методом наименьших квадратов. Остаточное среднеквадратичное отклонение временного ряда после вычитания двух основных гармоник оказалось равным $\sigma=0.716546 \mathrm{BT} / \mathrm{m}^{2}$.

Для отфильтрованного временного ряда реально наблюдаемой инсоляции были также рассчитаны автокорреляционная функция и спектр мощности $S(f)$. При выбранном максимальном сдвиге автокорреляции в 5000 отсчетов для использованного корреляционного окна Ханна ширина спектрального окна составляет $\Delta f=2.667 \times 10^{-4} \mathrm{~d}^{-1}$. Число степеней свободы спектральной оценки равно 5, а длина 90\%-ного логарифмического доверительного интервала 0.985 . График логарифма спектра мощности для рассматриваемых нами значений периодов $T>100^{\mathrm{d}}$ приведен на рис. 2, где вертикальным отрезком показан доверительный интервал. При построении графика использовался 1001 отсчет спектра.

Для полученного спектра характерно присутствие заметных флуктуаций, вызванных как погрешностью измерений потока, так и влиянием случайных изменений пло- 
щади фотосферных пятен и факельных полей, приводящих к вариации солнечной постоянной. Статистически значимым можно вые заподозрено Ригером и др. при изучении сильных солнечных вспышек, сопровождающихся мощным рентгеновским излуче-

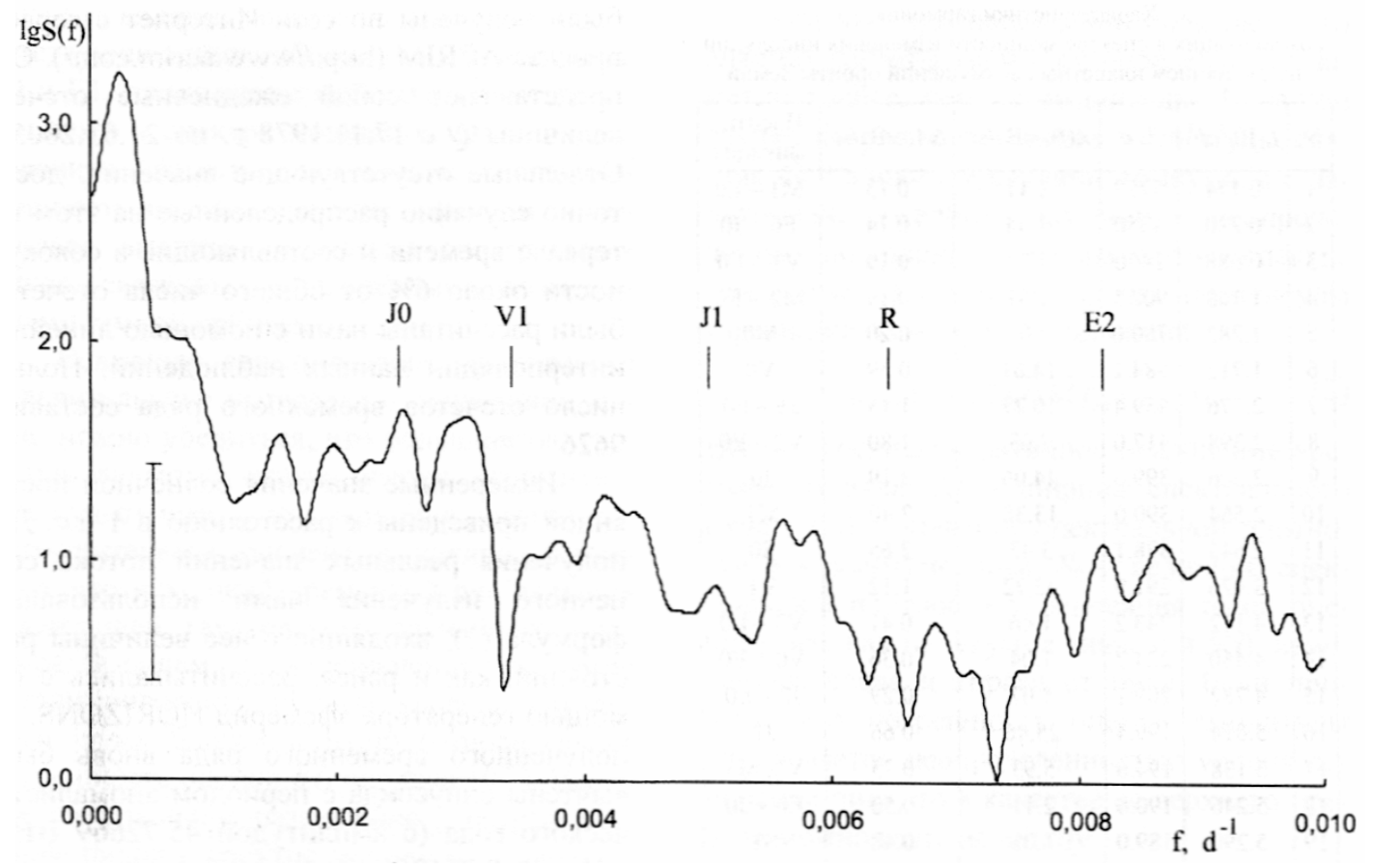

Рис. 2. График логарифма спектра мощности временного ряда реально наблюдаемого изменения инсоляции, вызванного планетными возмущениями орбиты Земли и вариацией солнечной постоянной

считать только пик спектра, расположенный вблизи начала координат. Он имеет период 11.4 года и соответствует основному циклу солнечной активности. Вследствие относительно небольшой длины ряда измерений солнечной постоянной спектральное разрешение оказывается в 18 раз меньше, чем на рис. 1. В результате расположенные близко друг к другу отдельные пики гармоник планетных возмущений сглаживаются и выглядят как один пик спектра. Наиболее мощные планетные гармоники, частоты которых на рис. 2 отмечены вертикальными отрезками, соответствуют заметным пикам в спектре ряда реальных изменений инсоляции. К сожалению, характер спектра не позволяет провести надежное измерение амплитуд гармоник.

Вертикальным отрезком с буквой $R$ на рис. 2 отмечена частота, соответствующая периоду $154^{\text {d. }}$ Существование цикла солнечной активности с этим периодом было впер- нием [16]. Позднее его наличие было подтверждено как по данным статистики вспышек [17], так и при анализе других индексов активности, включая блокирующую функцию пятен, поток на длине волны 10.7 см и числа Вольфа [18]. Присутствие заметного пика в спектре на данной частоте свидетельствует о том, что этот цикл проявляет себя и в изменении солнечной постоянной.

\section{Заключение}

Использование современных численных методов небесной механики и доступного программного обеспечения позволило дать ответ на вопрос о степени влияния короткопериодических составляющих планетных возмущений орбиты Земли на изменение величины потока солнечной радиации. В рассчитанном спектре мощности временного ряда инсоляции, наряду с гармониками годичного периода обращения Земли, четко проявляются гармоники синодических пе- 
риодов планет Венеры, Марса, Юпитера и Сатурна, а также гармоники, отвечающие комбинационным частотам. Периоды заметных планетных гармоник заключены в интервале от 100 дней до 15.7 года.

Основными гармониками спектра мощности являются две: гармоника J0, соответствующая синодическому периоду Юпитера $399^{\mathrm{d}} .0$, и первая гармоника синодического периода Венеры V1 с периодом 291 ${ }^{\mathrm{d}} .9$. Их амплитуды приблизительно равны и составляют около $40 \mathrm{mBT} / \mathrm{m}^{2}$. Следующая по мощности первая гармоника синодического периода Юпитера J1 имеет приблизительно в два раза меньшую амплитуду. Учитывая высокие возможности спектрального анализа, представляет большой интерес попытаться обнаружить проявление влияния этих гармоник во временных рядах метеорологических параметров.

Суммарное влияние гармоник, связанных с планетными возмущениями, характеризуется среднеквадратичным отклонением инсоляции около $0.1 \mathrm{BT} / \mathrm{m}^{2}$, что представляет собой достаточно заметную величину. Для сравнения: считающееся важным климатическим фактором изменение солнечной постоянной в ходе 11-летнего цикла имеет амплитуду около 1.0 Вт/м². Поэтому можно рекомендовать использовать в моделях палеоклимата и прогноза изменения климата реальные предвычисленные величины инсоляции, соответствующие точным значениям расстояния Земли от Солнца. Применение генератора эфемерид HORIZONS позволяет достаточно легко выполнять необходимые расчеты в интервале эпох от 3000 лет до н.э. до 3000 г. н.э.

Нами проанализирован также спектр мощности временного ряда реальных изменений инсоляции в 1978 - 2005 гг., вызванных как планетными возмущениями, так и вариацией солнечной постоянной. Показано, что наряду с основным пиком 11-летнего цикла солнечной активности, а также пиком с периодом $154^{\mathrm{d}}$, в этом спектре прослеживается и влияние планетных гармоник.

\section{Библиографический список}

1. White W.B., Lean J., Cayan D.R., Dettinger M.D. A response of global upper ocean temperature to changing solar irradiance // J. Geophys. Res. 1997. V.102, №C2. P.32553266.

2. Crowley T.J. Causes of climate change over past 1000 years // Science. 2000. V.289, №5477. P.270-277.

3. Reid G.C. Solar variability and the Earth's climate: introduction and overview // Space Science Reviews. 2000. V.94, №1-2. P.1-11.

4. Douglass D.H., Clader B.D. Climate sensitivity of the Earth to solar irradiance // Geophys. Res. Lett. 2002. V.29, №16. P.1786-1789.

5. Douglass D.H., Clader B.D., Knox R.S. Climate sensitivity of Earth to solar irradiance: update: Solar Radiation and Climate (SORCE) meeting on Decade Variability in the Sun and the Climate. Meredith, New Hampshire, 27-29 October, 2004. P.1-16 (http://arxiv.org/abs/physics/0411002).

6. Макарова Е.А., Харитонов А.В., Казачевская Т.В. Поток солнечного излучения. М., 1991. $400 \mathrm{c.}$

7. Willson R.C., Mordvinov A.V. Secular total solar irradiance trend during solar cycles 21-23 // Geophys. Res. Lett. 2003. V.30, №5. P.1199-2002.

8. Lee R.B., Wilson R.S., Thomas S. Long-term total solar irradiance (TSI) variability trends: 1984-2004: American Meteorological Society (AMS) 13th Conference on Satellite Meteorology and Oceanography, Norfolk, Virginia, 20-24 September 2004. Norfolk, Virginia, 2004. P6.31. P.1-5.

9. Миланкович М. Математическая климатология и астрономическая теория колебаний климата. М.; Л., 1939. 207 с.

10. Монин А.С. Вращение Земли и климат. Л., 1972. 112 с.

11. The solar output and its variation / Ed. O.R.White. Boulder, Colorado Associated University Press, 1977. 526 p.

12. Иванов В.В. Периодические колебания погоды и климата // Успехи физ. наук. 2002. Т.172, №7. С.777-811.

13. Нестеров В.В. Стандарт основных вычислений астрономии. М., 2001. $84 \mathrm{c}$.

14. Giorgini J.D., Yeomans D.K., Chamberlin A.B. et al. JPL's on-line Solar System data service // Bull. Amer. Astron. Soc. 1996. V.28, №3. P.1158.

15. Дженкинс Г., Ваттс Д. Спектральный анализ и его приложения. М., 1972. Т.1, 2. 603 с.

16. Rieger E., Kanbach G., Reppin C. et al. A 154-day periodicity in the occurrence of hard solar flares? // Nature. 1984. V.312. P.623-625.

17. Bai T., Sturrock P.A. Evidence for a fundamental period of the Sun and its relation to the 154 day complex of periodicities // Astrophys. J. 1993. V.409. P.476-486.

18. Lean J.L., Brueckner G.E. Intermediate-term solar periodicities: 100-500 days. // Astrophys. J. 1989. Vol.337. P.568578. 